\title{
Continuum and line emission of flares on red dwarf stars
}

\author{
E. Morchenko ${ }^{1}$-K. Bychkov ${ }^{1} \cdot$ M. Livshits ${ }^{2}$
}

\begin{abstract}
The emission spectrum has been calculated of a homogeneous pure hydrogen layer, which parameters are typical for a flare on a red dwarf. The ionization and excitation states were determined by the solution of steady-state equations taking into account the continuum and all discrete hydrogen levels. We consider the following elementary processes: electron-impact transitions, spontaneous and induced radiative transitions, and ionization by the bremsstrahlung and recombination radiation of the layer itself. The BibermanHolstein approximation was used to calculate the scattering of line radiation. Asymptotic formulae for the escape probability are obtained for a symmetric line profile taking into account the Stark and Doppler effects. The approximation for the core of the $\mathrm{H}-\alpha$ line by a gaussian curve has been substantiated.

The spectral intensity of the continuous spectrum, the intensity of the lines of the Balmer series and the magnitude of the Balmer jump have been calculated. The conditions have been determined for which the Balmer jump and the emission line intensities above the continuum decrease to such low values that the emission spectrum can be assumed to be continuum as well as the conditions at which the emission spectrum becomes close to the blackbody.
\end{abstract}

Keywords Red dwarfs, flares, Stark effect, $\mathrm{H}-\alpha$ line core, escape probability, emission spectrum

E. Morchenko

K. Bychkov

Sternberg Astronomical Institute, Moscow M.V. Lomonosov State University, 13, University Prospect, Moscow, Russia, 119992

M. Livshits

N.V. Pushkov Institute of Terrestrial Magnetism, Ionosphere and Radio Wave Propagation of Russian Academy of Sciences, 4, Kaluzhskoe Hwy, Troitsk, Moscow, Russia, 142190

\section{Introduction}

Gershberg \& Pikel'ner (1972) were the first who formulated the idea about the analogy of flares on red dwarf stars and on the Sun. At present it seems obvious that the physical nature of the activity of the Sun and red dwarf stars is similar (Gershberg (2005)).

Kostyuk \& Pikel'ner (1975) put forward the pioneering idea about the important role of gas-dynamic effects in the formation of the emission of solar flares. The authors showed that the radiative cooling of the shocked gas can be a source of the flare's emission in the optical range. The shock wave is produced by impulsive heating of the chromosphere by suprathermal particles.

The Kostyuk-Pikel'ner model predicts the formation of a relatively homogeneous gas layer, a few kilometers of thickness, which is responsible for the flare's optical emission in the continuum and spectral lines. The layer is located between the front of the downward shock and the region of plasma heated from above.

This model was applied to the problem of flares on red dwarf stars in Katsova et al. (1981). The authors fulfilled calculations of a one-dimensional radiative shock wave and demonstrated the formation of a region with a small gradient of the plasma parameters: temperature, pressure, and density (see Figures 3 and 4 in Katsova et al. (1981)). This result is confirmed by the calculations of the radiative cooling behind a shock front in the atmospheres of cool stars (see Fadeyev \& Gillet (2001) and Belova et al. (2014)) with a more detailed account for the elementary processes in the plasma: ionization, recombination, bremsstrahlung, excitation and de-excitation of discrete levels of atoms and ions as well as radiation scattering at the frequencies of spectral lines.

Red dwarf stars have a complex structure of flare spectra: the intensities of the emission lines strongly increase, the continuum emission is also enhanced; it 
is well approximated with Planck curves obtained for different temperatures at the blue and red sides relative to the Balmer jump (see Kowalski et al. (2013)). Layers producing the blackbody emission should be in a state close to thermodynamic equilibrium, whereas the gas generating strong emission lines is essentially non-equilibrium. Thus, the flare plasma includes at the same time equilibrium and non-equilibrium regions.

Therefore, we investigate the problem of the formation of an emission spectrum in a wide range of the cloud parameters. We consider a homogeneous gas layer of thickness $\mathcal{L}$ with definite values of temperature and density and consisting of pure hydrogen.

We develop the model of Drake \& Ulrich (1980) in three points. Firstly, we consider the influence of the layer's radiation (bremsstrahlung and recombination) on the occupation of atomic levels. This development is necessary, as the flare luminosity is stronger in the optical range than that of the quiescent atmosphere of the whole star. Secondly, we take into account that broadening of the high members of spectral series is caused exclusively by the linear Stark effect. Thirdly, we consider the two-temperature approximation for a flare layer.

In Section 1 the problem is formulated. In Section 2 we write the set of equations of ionization balance with regard to the continuum. In Section 3 the calculation of the continuum emission is described. In Section 4 we present the method of calculation of the quantum escape probability. In Section 5 the intensities of the emission spectral lines are calculated. Section 6 presents the results of the emission spectrum calculations of a layer with parameters possible in the flare regions of red dwarf stars.

\section{Statement of the Problem}

We calculate the emission in spectral lines and continuum of an homogeneous pure hydrogen layer. Its temperature, density and dimensions are assumed to be given. The value of the ion-atom temperature, $T_{a i}$, is taken to be greater than the value of the electron temperature, $T_{e}$, because both ions and atoms are heated more intensively than electrons at the shock front (see, e.g., Zel'dovich \& Raizer (1967)).

We use the results of the radiative shock wave calculations by Fadevev \& Gillet (2001) and Belova et al. (2014) to state the input parameter set. These calculations were fulfilled under the conditions of the atmosphere of a red giant star with an effective temperature of $T_{0}=3000 \mathrm{~K}$. The values of $T_{a i}$ and $T_{e}$ at the shock front are respectively $7-10 \mathrm{eV}$ and $\approx 0.7 \mathrm{eV}$.
The Lyman- $\alpha$ (Ly- $\alpha$ ) optical depth, $\tau_{12}$, in the cooling region, as it follows from Belova et al. (2014), lies in the range

$1 \cdot 10^{7} \leqslant \tau_{12} \leqslant 3 \cdot 10^{7}$.

The value of $\tau_{12}$ weakly depends on the density of the gas, as the cooling rate, $d S / d t$, is proportional to the hydrogen atom concentration, $N_{a}$. Thus, $N_{a}$ is cancelled:

$d \tau_{12} \propto N_{a} u \frac{d t}{d S} d S, \frac{d S}{d t} \propto N_{a} \Rightarrow d \tau_{12} \propto u d S$.

Here,

$S=T_{a i}+\frac{N_{e}}{N_{\mathrm{H}}} T_{e}$

$N_{e}$ is the electron density, $u$ is the velocity of the gas flowing from the shock front, and $d t$ is some time interval from the moment when a given gas layer intersects the shock front.

The value of $N_{\mathrm{H}}$ is equal to the summa of the proton and atom concentrations:

$N_{\mathrm{H}}=N_{p}+N_{a}$.

We accept $N_{\mathrm{H}}$ typical for the M-dwarf chromospheres:

$3 \cdot 10^{14} \mathrm{~cm}^{-3} \leqslant N_{\mathrm{H}} \leqslant 3 \cdot 10^{16} \mathrm{~cm}^{-3}$.

The layer thickness, $\mathcal{L}$, is defined to be such that the condition (1) is satisfied. The electron density is equal to

$N_{e}=N_{p}$.

The values of $T_{a i}$ and $T_{e}$ were obtained from a red giant star, which has a 100x smaller gravity compared to a red dwarf star. The ion-atom temperature at the shock front is determined by the shock velocity, $u_{0}$, and weakly depends on the density of the gas. If the value of $u_{0}$ lies in the range $50-70 \mathrm{~km} / \mathrm{sec}$, then it is true that

$6 \mathrm{eV} \leqslant T_{a i} \leqslant 12 \mathrm{eV}$.

The value of $T_{e}$ at the shock front is determined by the temperature $T_{0}$ of the unperturbed gas and heating due to the compression. The evolution of $T_{e}$ is caused by the balance between the supply of energy in elastic collisions with ions and atoms and radiative losses in the Lyman- $\alpha$ line. The radiative cooling in the hydrogen lines is the most intensive at $T_{e} \sim 1 \mathrm{eV}$ (see Belova et al. (2014)). Therefore, we assume that

$0.8 \mathrm{eV} \leqslant T_{e} \leqslant 1.5 \mathrm{eV}$. 
3 Balance Equations for the Populations of the Levels

The following processes we take into account: the electron impact ionization, excitation, and de-excitation, the triple recombination, radiative recombination (induced and spontaneous) as well as the influence of the layer's bremsstrahlung and recombination radiation on its ionization and excitation states.

The impact coefficients are taken from Johnson (1972). We use the Kramers approximation (see, e.g., Sobel'man (1963)) for the photoionization cross sections from the excited levels. The rates of the inverse processes are calculated following the detailed balance principle. The spectral lines optical depth can be rather large in this problem, so the scattering of line radiation should be taken into account. We introduce so called effective Einstein transition coefficients for the transitions between hydrogen levels $i$ and $k$ by the following formulae:

$\left\{\begin{array}{l}A_{k i}^{*} \\ B_{k i}^{*} \\ B_{i k}^{*}\end{array}\right\}=\left\{\begin{array}{l}A_{k i} \\ B_{k i} \\ B_{i k}\end{array}\right\} \times \theta_{i k}$.

Here, $\theta_{i k}$ is a photon escape probability out of the process of scattering. The values of $\theta_{i k}$ are calculated in the section 5 for the resonance as well as subordinate transitions.

According to the standard notation, $A_{k i}$ and $B_{k i}$ are respectively the probabilities of spontaneous and induced transition from the upper level $k$ to the lower level $i$, and $B_{i k}$ is the absorption coefficient.

We write the set of the balance equations for the discrete levels of a hydrogen atom:

$$
\begin{gathered}
\sum_{j=1}^{k-1} a_{1 j} N_{j}+a_{1 k} N_{k}+\sum_{j=k+1}^{n} a_{1 j} N_{j}=\alpha_{1} N_{e} N_{p}, \\
\sum_{j=1}^{k-1} a_{2 j} N_{j}+a_{2 k} N_{k}+\sum_{j=k+1}^{n} a_{2 j} N_{j}=\alpha_{2} N_{e} N_{p}, \\
\ldots \ldots \ldots \ldots \ldots \\
\sum_{j=1}^{k-1} a_{k j} N_{j}+a_{k k} N_{k}+\sum_{j=k+1}^{n} a_{k j} N_{j}=\alpha_{k} N_{e} N_{p}, \\
\ldots \ldots \ldots{ }_{j=k+1}^{n} a_{n j} N_{j}=\alpha_{n} N_{e} N_{p},
\end{gathered}
$$

where

$$
\alpha_{i}=r_{i}+\gamma_{i} N_{e}, \quad i=1, \ldots, n .
$$

We assume that the maximum value of the principal quantum number, $n$, is equal to an effective series limit. It can be evaluated by the Inglis-Teller formula Vidal $(1966))$, as

$\lg n=3.10-0.13 \lg \left(2 N_{e} / \mathrm{cm}^{-3}\right)$.

The following designations are introduced:

$N_{k}$ — the density number of the hydrogen atoms on the discrete level with the principal quantum number $k$;

$r_{k}, \gamma_{k}$ - the radiative and triple recombination coefficients on the $k$-th level, respectively;

$a_{i k}(i \neq k)$ - the transition rate from the level $k$ to the level $i$;

$a_{k k}$ - the escape rate from the level $k$.

We write the explicit formulae for the matrix coefficients in the left side of the equation set.

Excitation rate $(k<i)$ is:

$a_{i k}=-\left[q_{k i}\left(T_{e}\right) N_{e}+4 \pi I_{k i} B_{k i}^{*}\right]$,

where

$q_{k i}\left(T_{e}\right)$ is the electron impact excitation;

$I_{k i}$ is the spectral intensity of the layer continuous radiation in the frequency of $k \leftrightarrow i$ transition.

Deactivation rate $(k>i)$ is:

$a_{i k}=-\left[q_{k i}\left(T_{e}\right) N_{e}+A_{k i}^{*}+4 \pi I_{k i} B_{k i}^{*}\right]$,

where $q_{k i}\left(T_{e}\right)$ is the impact deactivation coefficient.

The diagonal elements are

$$
\begin{aligned}
& a_{k k}=\sum_{j=1}^{k-1}\left[q_{k j}\left(T_{e}\right) N_{e}+A_{k j}^{*}+4 \pi I_{k j} B_{k j}^{*}\right]+ \\
& +\sum_{j=k+1}^{n}\left[q_{k j}\left(T_{e}\right) N_{e}+4 \pi I_{k j} B_{k j}^{*}\right]+\mathcal{P}_{k}+q_{k} \cdot N_{e}
\end{aligned}
$$

where $q_{k}$ is the electron impact ionization coefficient, and $\mathcal{P}_{k}$ is the photoionization rate from the level $k$ :

$\mathcal{P}_{k}=4 \pi \int_{\nu_{k}}^{\infty} \frac{I_{\nu}}{h \nu} \sigma_{k}(\nu) d \nu$.

Here, $\nu_{k}$ is the photoelectric threshold from level $k, h$ is the Plank constant, $\sigma_{k}(\nu)$ is the cross section in the Kramers approximation:

$\sigma_{k}(\nu)=\frac{64}{3 \sqrt{3}} \alpha \pi a_{0}^{2} \cdot k^{-5} \cdot\left(\frac{\mathrm{Ry}}{h \nu}\right)^{3}$,

where $\alpha$ is the fine structure constant, $a_{0}$ is the Bohr radius.

The spontaneous recombination coefficient, $r_{k}^{(s)}$, can be 
calculated from (17) using the principle of detailed balance:

$$
\begin{array}{rlrl}
r_{k}^{(s)} & =\mathcal{R}\left(\beta_{k}\right) E_{1}\left(\beta_{k}\right), & \beta_{k} & =\frac{\mathrm{Ry}}{k^{2} T_{e}} \\
\mathcal{R}(x) & =C_{r} \cdot x^{3 / 2} e^{x}, & C_{r}=\frac{2^{6}}{3} \sqrt{\frac{\pi}{3}} \alpha^{4} c a_{0}^{2},
\end{array}
$$

where $E_{1}(x)$ is the exponential integral of order 1 .

The coefficients of induced recombination, $r_{k}^{(\text {ind })}$, is given in the next section. It is true that

$r_{k}=r_{k}^{(s)}+r_{k}^{(\text {ind })}$

Summing the set (10) over all discrete levels yields the identity

$\sum_{i}\left(\mathcal{P}_{i}+q_{i} N_{e}\right) \cdot N_{i}=\sum_{i}\left(r_{i}+\gamma_{i} N_{e}\right) \cdot N_{e} N_{p}$

As a result, we come to the set of homogeneous equations with singular matrix for the relative level occupations, $y_{i}=N_{i} / N_{a}$ :

$\sum_{j=1}^{n} \tilde{a}_{i j} y_{j}=0, i=1, \ldots, n$.

where

$$
\begin{aligned}
\tilde{a}_{i k} & =a_{i k}-\frac{\alpha_{i}}{\Upsilon}\left(\mathcal{P}_{k}+q_{k} N_{e}\right), \\
\alpha_{i} & =r_{i}+\gamma_{i} N_{e}, \quad \Upsilon=\sum_{j=1}^{n} \alpha_{j} .
\end{aligned}
$$

To avoid the singularity, the last equation $(k=n)$ is replaced by the normalization condition

$$
\sum_{k=1}^{n} y_{k}=1
$$

We take into account the scattering of the ionizing radiation using the division of $r_{k}$ by $1+\tau_{k}$, where $\tau_{k}$ is the optical depth on the photoionization threshold from the level $k$.

\section{Continuum Emission}

Continuum emission consists of two components: freefree and bound-free. The following formula is valid for an homogeneous layer:

$I_{\nu}=S_{\nu} \cdot\left(1-e^{-\tau_{\nu}}\right)$,

where $S_{\nu}$ is the source function, which is equal to the ratio of the emission coefficient, $\varepsilon_{\nu}$, to the absorption coefficient, $\varkappa_{\nu}$ :

$S_{\nu}=\frac{\varepsilon_{\nu}}{\varkappa_{\nu}}=\frac{\varepsilon_{\nu}^{(b)}+\sum_{j=k}^{n} \varepsilon_{\nu j}^{(r)}}{\varkappa_{\nu}^{(b)}+\sum_{j=k}^{n} \varkappa_{\nu j}^{(i)}}$.

The summation is over all the levels for which the frequency of the photoelectric threshold is less than $\nu$ :

$h \nu \geqslant \frac{\mathrm{Ry}}{k^{2}}$.

The Menzel factor is designated by $b_{k}$ :

$b_{k}=\frac{N_{k}}{N_{e} N_{p}} \frac{\left(2 \pi m_{e} T_{e}\right)^{3 / 2}}{k^{2} h^{3}} \exp \left(-\beta_{k}\right)$.

It shows the deviation of the level population from its equilibrium value. This factor is equal to 1 when excitation and ionization are described by the formulae of Boltzmann and Saha.

We use the Kramers approximation for the absorption coefficients (Sobel'man (1963), §34.4):

$\varkappa_{\nu}^{(b)}=\frac{2^{8}}{3 \sqrt{3}} \alpha \pi^{5 / 2} a_{0}^{5} \sqrt{\frac{\mathrm{Ry}}{T_{e}}}\left(\frac{\mathrm{Ry}}{h \nu}\right)^{3}\left(1-e^{-y}\right) N_{e} N_{p}$,

$\begin{aligned} \varkappa_{\nu k}^{(i)}= & \frac{2^{9}}{3 \sqrt{3}} \alpha \pi^{5 / 2} a_{0}^{5} \frac{1}{k^{3}} b_{k}\left(\frac{\mathrm{Ry}}{h \nu}\right)^{3}\left(\frac{\mathrm{Ry}}{T_{e}}\right)^{3 / 2} \times \\ & \times\left(1-\frac{1}{b_{k}} e^{-y}\right) e^{\beta_{k}} N_{e} N_{p}, \quad y \geqslant \beta_{k},\end{aligned}$

where $y=\frac{h \nu}{T_{e}}$.

We write the relation between the ionization coefficient from the level $k, \varkappa_{\nu k}^{(i)}$, and the bremsstrahlung coefficient, $\varkappa_{\nu}^{(b)}$ :

$\frac{\varkappa_{\nu k}^{(i)}}{\varkappa_{\nu}^{(b)}}=\varphi_{k} \frac{b_{k}-e^{-y}}{1-e^{-y}}, \quad \varphi_{k} \equiv \frac{2}{k} \beta_{k} e^{\beta_{k}}$.

The value of $\tau_{\nu}$ is equal to the sum of bremsstrahlung optical depth, $\tau_{\nu}^{(b)}$, and photoionization one, $\tau_{\nu}^{(i)}$ :

$\tau_{\nu}=\tau_{\nu}^{(b)}+\tau_{\nu}^{(i)}=\left(\varkappa_{\nu}^{(b)}+\sum_{j=k}^{n} \varkappa_{\nu j}^{(i)}\right) \cdot \mathcal{L}$.

The free-free absorption and emission coefficients are related by Kirchhoff low:

$\frac{\varepsilon_{\nu}^{(b)}}{\varkappa_{\nu}^{(b)}}=B_{\nu}\left(T_{e}\right)$ 
The Menzel factor connects the radiative recombination coefficient, $\varepsilon_{\nu k}^{(r)}$, and photoionization coefficients for the $k$-th level:

$\frac{\varepsilon_{\nu k}^{(r)}}{\varkappa_{\nu k}^{(i)}}=B_{\nu}\left(T_{e}\right) \cdot \frac{1-e^{-y}}{b_{k}-e^{-y}}, \quad\left(y \geqslant \beta_{k}\right)$.

Using the last formula and taking into account (27), we can transform (26):

$$
\begin{gathered}
S_{\nu}=\frac{\varepsilon_{\nu}^{(b)} / \varkappa_{\nu}^{(b)}+\sum_{j=k}^{n}\left(\varepsilon_{\nu j}^{(r)} / \varkappa_{\nu j}^{(i)}\right) \cdot\left(\varkappa_{\nu j}^{(i)} / \varkappa_{\nu}^{(b)}\right)}{1+\sum_{j=k}^{n} \varkappa_{\nu j}^{(i)} / \varkappa_{\nu}^{(b)}}= \\
=B_{\nu}\left(T_{e}\right) \cdot \frac{1+\sum_{j=k}^{n} \frac{\varkappa_{\nu j}^{(i)}}{\varkappa_{\nu}^{(b)}} \cdot \frac{1-e^{-y}}{b_{j}-e^{-y}}}{1+\sum_{j=k}^{n} \frac{\varkappa_{\nu j}^{(i)}}{\varkappa_{\nu}^{(b)}}}=B_{\nu}\left(T_{e}\right) \cdot \psi_{k}(y),
\end{gathered}
$$

where the following function $\psi_{k}$ is introduced:

$$
\psi_{k}(y) \equiv \frac{1+\sum_{j=k}^{n} \varphi_{j}}{1+\frac{1}{1-e^{-y}} \sum_{j=k}^{n} \varphi_{j} \cdot\left(b_{j}-e^{-y}\right)} .
$$

The induced recombination coefficient is equal to

$$
r_{k}^{(\mathrm{ind})}=\mathcal{R}\left(\beta_{k}\right) \cdot \int_{\beta_{k}}^{\infty} \frac{\psi_{k}(y) \cdot\left(1-e^{-\tau_{\nu}}\right)}{e^{y}-1} \frac{e^{-y}}{y} d y .
$$

As is easily seen from (29), the continuum optical depth, $\tau_{\nu}$, is the function of $y$ when the frequency is varied and the other parameters are unchanged.

\section{Photon escape probability}

The source of optical emission moves slowly and cohesively, i.e. there is no noticeable velocity gradient. Therefore, the Biberman-Holstein approximation (Holstein (1947)) is used to calculate the scattering of line radiation. Consider a stationary gas that occupies a volume $V$ bounded by a closed surface $S$. Let $\mathbf{r}$ is the radius-vector of a point within the volume. According to Biberman et al. (1982), the photon escape probability from this point outside the homogeneous plasma can be written in the form:

$\theta(\mathbf{r})=\int d \omega a_{\omega} \oint_{S} \frac{(\mathbf{d} \mathbf{S}, \mathbf{R}-\mathbf{r})}{4 \pi|\mathbf{R}-\mathbf{r}|^{3}} \exp \left(-k_{\omega}|\mathbf{R}-\mathbf{r}|\right)$.
Here, $\omega$ is the angular frequency, $a_{\omega}$ is the spectral line profile, and $k_{\omega}$ is the line absorption coefficient. $\mathbf{R}$ denotes the radius-vector of a point belonging to the surface $S$.

Let the gas occupies a layer of finite thickness $\mathcal{L}$. In this case, the mean escape probability is equal to

$\theta=\int d \omega a_{\omega} \mathcal{G}\left(\tau_{\omega}\right)$

where

$\mathcal{G}\left(\tau_{\omega}\right)=\frac{1}{4 \tau_{\omega}}\left[1-2 E_{3}\left(2 \tau_{\omega}\right)\right], \quad \tau_{\omega}=\frac{k_{\omega} \mathcal{L}}{2}$.

In these expressions, $\tau_{\omega}$ is the optical depth at the center of the layer and $E_{3}(x)$ is the third-order exponential integral (see, e.g., Smirnov (1972)).

As stated in Sobolev \& Grinin (1996), the very wide wings of the Balmer lines in the optically thick flare plasma are due to the linear Stark effect. When the Stark broadening by electrons and ions is treated in a semi-classical picture, the static limit is valid in the distant long-wavelength line wing (Sobel'man (1963), $\S 38.1)$ :

$a_{\omega}^{\mathrm{St}} d \omega \approx \frac{15}{4 \sqrt{2 \pi}} \frac{\left(\mathcal{B}_{k n^{\prime}} \mathcal{E}_{0}\right)^{3 / 2}}{\Delta \omega_{k n^{\prime}}^{5 / 2}} d \omega$.

Here, $\mathcal{B}_{k n^{\prime}}$ is the Stark broadening parameter of the transition from level $k$ to level $n^{\prime}\left(k>n^{\prime}\right), \mathcal{E}_{0}$ is the normal Holtsmark field, and $\Delta \omega_{k n^{\prime}}=\omega-\omega_{k n^{\prime}}, \omega_{k n^{\prime}}$ is the transition angular frequency. The Stark width is approximately equal to

$\mathcal{B}_{k n^{\prime}} \mathcal{E}_{0}=\left(\frac{\pi}{2}\right)^{2 / 3} \frac{\hbar}{m_{e}}\left(k^{2}-n^{\prime 2}\right) N_{p}^{2 / 3}$.

The spectral line profile is determined by radiation damping, Doppler effect and pressure effects. The extensive tables of the Stark broadened profiles for hydrogen plasma at $T_{a i}=T_{e}$ have been published by Stehle (1994). In the case of $T_{a i}>T_{e}$ we will use a simple model of the line profile with the Doppler core and the Holtsmark wings.

We consider, e.g., the $\mathrm{H}-\alpha$ line profile in order to justify this model. The following set of parameters is assumed:

$N_{e}=10^{14} \mathrm{~cm}^{-3}, T_{a i}=6 \mathrm{eV}, T_{e}=1 \mathrm{eV}$.

We introduce the value of $\Omega_{32}$ such that

$\Omega_{32}=v^{2}\left[6 \frac{e^{2} a_{0}}{\hbar}\right]^{-1}$, where $v$ is the hydrogen atom 
velocity (along a line of sight). The value of $v$ can be evaluated as

$v \sim \sqrt{\frac{3 T_{a i}}{m_{H}}} \Rightarrow \Omega_{32} \sim 2.87 \cdot 10^{12} \mathrm{sec}^{-1}$.

Here, $m_{H}$ is the hydrogen atom mass. The Doppler width, $\Delta \omega_{k n^{\prime}}^{D}$, is equal to

$\Delta \omega_{k n^{\prime}}^{D}=\omega_{k n^{\prime}} \sqrt{\frac{2 T_{a i}}{m_{H} c^{2}}}$.

Here, $k=3, n^{\prime}=2 \Rightarrow \Delta \omega_{32}^{D} \approx 3.246 \cdot 10^{11} \mathrm{sec}^{-1}$ and $\Delta \omega_{32}^{D} \ll \Omega_{32}$. Therefore, the static limit (39) can be used when $\Delta \omega_{32} \gg \Omega_{32}$, i.e., in the line wing (see Sobel'man (1963), §38.1, §36.6).

On the other hand, we introduce the parameter $\gamma$, which is equal to the sum of the radiative damping constant, $\gamma_{\mathrm{rad}}$, and the parameter $\gamma_{2}$ :

$\gamma=\gamma_{\mathrm{rad}}+\gamma_{2} ; \gamma_{2} \sim 72 \pi^{3} N v^{-1} \sim 5.4 \cdot 10^{-4} N$

where $N$ is the concentration of perturbing particles. $\gamma_{\mathrm{rad}}<2 \cdot 4.67 \cdot 10^{8} \mathrm{sec}^{-1} \Rightarrow \gamma \sim \gamma_{2}$. If $N \sim N_{e}$ then $\gamma /\left(\Delta \omega_{32}^{D}\right) \ll 1$. Therefore, the central part of the $\mathrm{H}-\alpha$ line profile is the Doppler profile (see Sobel'man (1963), $\S 36.6)$.

In general, there is some analogy between the line profile with the Doppler core and the Holtsmark wings and the Voigt profile when the Voigt parameter $a$ is much less than 1. The normalized Voigt function is

$a_{\omega}^{V}=\frac{1}{\sqrt{\pi} \Delta \omega_{k n^{\prime}}^{D}} H(a, v)$,

where $v$ is the dimensionless frequency displacement from the line center in units of $\Delta \omega_{k n^{\prime}}^{D}$. For $a \ll 1$ it can be assumed that in the first approximation (see, e.g., Ivanov (1969))

$H(a, v)= \begin{cases}\exp \left(-v^{2}\right), & 0 \leq|v| \leq\left|v_{0}\right| \\ \frac{a}{\sqrt{\pi} v^{2}}, & |v| \geq\left|v_{0}\right| .\end{cases}$

The value of $\left|v_{0}\right|$ is the solution of the equation

$\exp \left(-v_{0}^{2}\right)=\frac{a}{\sqrt{\pi} v_{0}^{2}}$

The transition from the Doppler core to the Lorentz wings in the Voigt profile is quite sharp Mihalas $(1974))$.

\subsection{Ly- $\alpha$ line}

The following model symmetric profile is similar to the Voigt profile (45):

$a_{\omega}=\frac{C}{\sqrt{\pi} \Delta \omega_{k n^{\prime}}^{D}} \Phi\left(b_{k n^{\prime}}, v\right)$,

where $C$ is a normalizing constant. The function $\Phi$ is defined by

$\Phi\left(b_{k n^{\prime}}, v\right)= \begin{cases}\exp \left(-v^{2}\right), & 0 \leq|v| \leq\left|v_{0}\right| \\ \frac{15}{4 \sqrt{2}} \cdot \frac{b_{k n^{\prime}}}{v^{5 / 2}}, & |v| \geq\left|v_{0}\right| .\end{cases}$

In these expressions, the value of parameter $b_{k n^{\prime}}$ is significantly less than unity:

$b_{k n^{\prime}} \equiv\left(\frac{\mathcal{B}_{k n^{\prime}} \mathcal{E}_{0}}{\Delta \omega_{k n^{\prime}}^{D}}\right)^{3 / 2} \ll 1$.

We can find the constant $C$ from the equations

$1=\int_{-\infty}^{+\infty} a_{\omega} d \omega=C \cdot\left(\operatorname{erf} v_{0}+\frac{5}{\sqrt{2 \pi}} \cdot \frac{b_{k n^{\prime}}}{v_{0}^{3 / 2}}\right)$

$\frac{15}{4 \sqrt{2}} \cdot \frac{b_{k n^{\prime}}}{v_{0}^{5 / 2}}=\exp \left(-v_{0}^{2}\right)$.

Drake \& Ulrich (1980) introduced the model profile phenomenologically. Here, we give the theoretical foundation for its application.

To make an estimate of the optical depth in the Ly- $\alpha$ line at $|v|=\left|v_{0}\right|$, we use (41). According to (40), the Stark width of the $\mathrm{Ly}-\alpha$ line is approximately equal to $\mathcal{B}_{21} \mathcal{E}_{0}=1,01 \cdot 10^{10} \mathrm{sec}^{-1}$, the Doppler width is $\Delta \omega_{21}^{D}=$ $1.75 \cdot 10^{12} \mathrm{sec}^{-1}$; whence it follows that

$b_{21}=4.385 \cdot 10^{-4} \ll 1$.

Thus, the approximation (48) is adequate in our problem. Using (50) and (51), we find that $\left|v_{0}\right| \approx 3.095$ and $C=1$. If the optical depth at the line center is equal to $\tau_{\omega_{0}}^{D}=3 \cdot 10^{7}$, then it is true that

$\tau_{\omega}\left(\left|v_{0}\right|\right)=\tau_{\omega_{0}}^{D} \cdot \exp \left(-v_{0}^{2}\right)=2 \cdot 10^{3} \gg 1$.

Therefore, the Ly- $\alpha$ photon lives the medium at the frequencies of the distant line wings.

As a result, equation (37) can be written in the form:

$\theta_{12}=b_{21} \frac{15}{\sqrt{8 \pi}} \int_{0}^{+\infty} \mathcal{G}\left(\tau_{\omega}\right) \frac{d v}{v^{5 / 2}} ; \quad \tau_{\omega}=\tau_{\omega}(v)$. 
It is obvious that

$k_{\omega}=k_{\omega_{0}}^{D} \frac{15}{4 \sqrt{2}} \cdot \frac{b_{21}}{v^{5 / 2}}, \quad \tau_{\omega}=\tau_{\omega_{0}}^{D} \frac{15}{4 \sqrt{2}} \cdot \frac{b_{21}}{v^{5 / 2}}$.

Here, $k_{\omega_{0}}^{D}$ is the absorption coefficient at the Doppler profile center:

$k_{\omega_{0}}^{D}=\frac{2 \pi^{2} e^{2}}{m_{e} c} f_{n^{\prime} k} N_{n^{\prime}} \frac{1}{\sqrt{\pi} \Delta \omega_{k n^{\prime}}^{D}} ; \quad \tau_{\omega_{0}}^{D}=\frac{k_{\omega_{0}}^{D} \mathcal{L}}{2}$,

$f_{n^{\prime} k}$ is the oscillator strength for absorption $\left(n^{\prime}=1\right.$, $k=2$ ). Taking into account that $\tau_{\omega_{0}}^{D} \gg 1$, we obtain the asymptotic formula for the mean escape probability:

$\theta_{12}=\frac{4}{5} \cdot\left(\frac{15}{4 \sqrt{2}}\right)^{2 / 5} \frac{b_{21}^{2 / 5}}{\sqrt{\pi}\left(\tau_{\omega_{0}}^{D}\right)^{3 / 5}} \int_{0}^{\tau_{\omega_{0}}^{D} \rightarrow+\infty} \mathcal{G}\left(\tau_{\omega}\right) \frac{d \tau_{\omega}}{\tau_{\omega}^{2 / 5}}$.

Consider the integral

$I=\int_{0}^{+\infty} \mathcal{G}(\tau) \frac{d \tau}{\tau^{\kappa}}=\frac{1}{4} \int_{0}^{+\infty}\left[1-2 E_{3}(2 \tau)\right] \frac{d \tau}{\tau^{1+\kappa}}, \quad \kappa \in(0,1)$

By using the formula of integration by parts, this equation can be represented in the form:

$I-\frac{1}{\kappa} \int_{0}^{+\infty} E_{2}(2 \tau) \frac{d \tau}{\tau^{\kappa}}=-\left.\frac{1}{4 \kappa} \frac{\left[1-2 E_{3}(2 \tau)\right]}{\tau^{\kappa}}\right|_{0} ^{+\infty}$.

If the value of $\kappa$ lies between 0 and 1 , then the r.h.s. of the last equation tends to zero. Thus, we obtain that

$I=\frac{\mu^{2}}{2 \mu-1} \frac{\Gamma(1-1 / \mu)}{2^{1-1 / \mu}}, \quad \mu \equiv \kappa^{-1}$,

where $\Gamma$ is the gamma function. In this problem, the value of $\mu$ is equal to $5 / 2$. Finally, we come to

$\theta_{12} \approx\left(\frac{\mathcal{B}_{21} \mathcal{E}_{0}}{\Delta \omega_{21}^{D}}\right)^{3 / 5} \frac{1}{\left(\tau_{\omega_{0}}^{D}\right)^{3 / 5}}$

The exponent $3 / 5$ in the last equation is the feature of the linear Stark effect.

\subsection{Generalization for the case of a multilevel atom}

Strictly speaking, the resulting expression for $\theta_{12}$ is valid only for a model two-level atom. However, the escape probability approach can be applied also for a multilevel atom. We will use the model profile (48) up to $\bar{b}=3 \cdot 10^{-2}$ (for more details, see Stehle (1994)).
Then the principal quantum numbers, $k$ and $n^{\prime}$, must satisfy the condition

$\bar{b} \geqslant b_{k n^{\prime}}=\left(\frac{k^{2} n^{\prime 2}}{4}\right)^{3 / 2} b_{21}$

Constant $C$ is approximately unity in the range $10^{-4} \leqslant$ $b_{k n^{\prime}} \leqslant 10^{-2}$; therefore, it is true that

$\theta_{n^{\prime} k} \approx\left(\frac{\mathcal{B}_{k n^{\prime}} \mathcal{E}_{0}}{\Delta \omega_{k n^{\prime}}^{D}}\right)^{3 / 5} \frac{1}{\left(\tau_{\omega_{0}}^{D}\right)^{3 / 5}}$,

where $\tau_{\omega_{0}}^{D}$ is the optical depth in the $n^{\prime} \rightarrow k$ transition .

Since the Stark width increases with increasing $k$, the Stark broadening dominates over the Doppler broadening in the limit $k \gg 1$. Then we use the following model profile:

$a_{\beta}= \begin{cases}a_{0}, & 0 \leq|\beta| \leq\left|\beta_{0}\right| \\ \frac{3}{2} \frac{1}{\beta^{5 / 2}}, & |\beta| \geq\left|\beta_{0}\right|,\end{cases}$

Here, $a_{0}$ is some constant, $\beta \equiv \frac{\omega-\omega_{0}}{\mathcal{B}_{k n^{\prime}} \mathcal{E}_{0}}$. The normalization condition gives

$\beta_{0} \approx 3, a_{\omega_{0}} \approx \frac{0.1}{\mathcal{B}_{k n^{\prime}} \mathcal{E}_{0}}$

After some calculations we obtain that

$\theta_{n^{\prime} k} \approx \frac{0.36}{\tau_{\omega_{0}}^{3 / 5}}, \tau_{\omega_{0}}=\frac{\pi^{2} e^{2}}{m_{e} c} f_{n^{\prime} k} N_{n^{\prime}} a_{\omega_{0}} \mathcal{L}$.

5.3 Application to the observations of the $\mathrm{H}-\alpha$ line profile

It is interesting to note that the profile with the Doppler core and the Stark-broadened wings is similar to the $\mathrm{H}$ $\alpha$ line profile in the flare on UV Ceti (dM5.6e) published in Eason et al. (1992). The authors found a Doppler profile to fit the line core: for $\mathrm{H}-\alpha$ during outburst, the Doppler width was equal to $0.9 \AA$. Thus, it is true that $T_{a i} \approx 8.8 \mathrm{eV}$ and $\Delta \omega_{32}^{D} \approx 3.93 \cdot 10^{11} \mathrm{sec}^{-1}$. Further, Eason et al. found that $\log \left(N_{e}\right)=14.75 \Rightarrow B_{32} \mathcal{E}_{0} \approx$ $5.33 \cdot 10^{10} \mathrm{sec}^{-1}$ and $\gamma \sim 2.5 \cdot 10^{11} \mathrm{sec}^{-1}$. Thus, we obtain that $\gamma / \Delta \omega_{32}^{D} \sim 0.6$ and $b_{32} \ll 1$.

The authors satisfactorily represented part of the long-wavelength wing with a Stark profile from the paper by Underhill \& Waddell (1959). In this paper the Stark profile was calculated taking into account only the ion broadening in context of the Holtsmark distribution of the electric microfields, while the Stark broadening by electrons was neglected. But as stated in 
Sobolev \& Grinin (1995), the electron broadening dominates in stellar flares. We consider that the deviation of the observed $\mathrm{H} \alpha$ line profile from the Stark profile in the red wing (see Fig. 9 in Eason et al. (1992)) is almost entirely due to neglecting the contribution of electrons to the line broadening in the optically thick flare plasma.

\section{Spectral Line Emission}

To simplify the notation, we have in mind the Balmer series. The intensity of the emission in the spectral line frequencies, $I_{\nu}^{(k)}$, is equal to

$$
I_{\nu}^{(k)}=S_{\nu}^{(k)} \cdot\left[1-e^{-\tau_{\nu}^{(k)}-\tau_{\nu}}\right]
$$

where index $k$ identifies the upper level of the transition $k \rightarrow 2$. We have denoted by $\tau_{\nu}^{(k)}$ the optical depth in the frequencies of the $2 \rightarrow k$ transition; the negative absorption being taken into account:

$\tau_{\nu}^{(k)}=\varkappa_{0}^{(k)} \cdot a_{\nu} \cdot \mathcal{L}$.

Here,

$\varkappa_{0}^{(k)}=\frac{\pi e^{2}}{m_{e} c} f_{2 k} \cdot N_{2}\left[1-\frac{b_{k}}{b_{2}} \exp \left(-\frac{E_{k 2}}{T_{e}}\right)\right]$,

The spectral line intensity, $I_{k}$, is equal to the integral of (60) over the line profile:

$I_{k}=\int S_{\nu}^{(k)} \cdot\left[1-e^{-\tau_{\nu}^{(k)}-\tau_{\nu}}\right] d \nu$.

In this section, we consider that the continuum absorption is negligible within the profile of a spectral line. So, we write the expression for the source function taking into account only the line emission and absorption:

$S_{\nu}^{(k)}=B_{\nu}\left(T_{e}\right) \cdot \frac{1-e^{-y}}{b_{2} / b_{k}-e^{-y}}$.

The Planck function varies very slowly within the line profile. Therefore, the source function at the frequency of the line center, $S_{k}$, can be taken outside the integral sign:

$I_{k} \approx S_{k} \cdot \int_{\nu_{l}}^{\nu_{u}}\left[1-e^{-\tau_{\nu}\left(\nu_{k}\right)} e^{-\tau_{\nu}^{(k)}}\right] d \nu$.

For the same reason, we consider that the continuum optical depth within the line profile is equal to $\tau_{\nu}\left(\nu_{k}\right)$. The limits of integration are the frequencies where the line emission merges into continuum. They are defined by the following condition:

$\tau_{\nu}^{(k)}\left(\nu_{l}\right)=\tau_{\nu}^{(k)}\left(\nu_{u}\right)=\tau_{\nu}\left(\nu_{k}\right)$.

The simplified formulae (65) and (66) do not introduce significant inaccuracies in $I_{k}$, as the transition region is quite narrow. Moreover, the emission intensity in this region is much lower than the intensity in the line core.

Note that there are some factors simplifying the calculation of integral (65). If the condition (10) is satisfied, then the central optical depth, $\tau_{k}$, is rather large. Consequently, the intensity in the line core frequencies coincides with a source function.

In those parts of the line profile where $\tau_{k}$ is not too large, we use for $a_{\nu}$ a simple approximation (58), assuming that for the frequency displacement up to $\beta_{0}$, the layer remains opaque. We introduce frequency displacement $\beta_{0} \geqslant 3$ which satisfies the condition

$\tau_{\nu}^{(k)}>5$

In the offset range

$0 \leq|\beta| \leq\left|\beta_{0}\right|$

it is assumed that the intensity is equal to the source function, and in the line wings we calculate the integral

$D_{k}=2 \int_{\beta_{0}}^{\beta_{u}}\left(1-e^{-\tau_{\nu}\left(\nu_{k}\right)} \cdot e^{-\tau_{k} \beta^{-5 / 2}}\right) d \beta$.

Here, parameter $\tau_{k}$ is defined as

$\tau_{k}=\varkappa_{0}^{(k)} \cdot \frac{3}{2} \cdot \frac{2 \pi}{\mathcal{B}_{k 2} \mathcal{E}_{0}} \cdot \mathcal{L}$,

and the upper limit is calculated according to (66). Thus, the total energy emitted in the line is

$I_{k}=S_{k} \cdot\left(2 \beta_{0}+D_{k}\right) \cdot \frac{\mathcal{B}_{k 2} \mathcal{E}_{0}}{2 \pi}$

where the Stark width, $\mathcal{B}_{k 2} \mathcal{E}_{0}$, is determined by formula (40).

\section{Results}

We performed calculations of the spectral line and continuum intensity for different values of density and temperature of a homogeneous gas layer under the conditions of flares on red dwarf stars. The layer thickness $\mathcal{L}$ is fixed. We calculated the optical depth at the center of the Balmer series lines (see table 1) and the Menzel factors; in all cases the values of $b_{k}$ do not differ from 
unity (see table 2). It is important that there is no external source of radiation ionizing the hydrogen atom from the excited levels.

Let us address the results of calculations of the emission spectrum. Figure 1 shows how the emission lines are "immersed" in a continuum with increasing density of the gas. The horizontal axis represents the wavelength $\lambda$ in Angströms, vertical - the logarithm of the ratio $I_{\nu} / B_{\nu}$. Balmer lines are indicated by vertical line segments, its height is proportional to the line center intensity. In all the graphs, the following values of the parameters are assumed: $T_{e}=1.2 \mathrm{eV}, T_{a i}=6 \mathrm{eV}$, and $\mathcal{L}=20 \mathrm{~km}$. The intensity of the emission at the centers of the Balmer series lines is very close to the Planck function at the electron temperature $B_{\lambda}\left(T_{e}\right)$. Therefore, all the spectral lines have the same upper boundary corresponding to zero on the vertical axis. As the rise of the continuum occurs, first $\mathrm{H}_{\alpha}$ "immerses", then $\mathrm{H}_{\beta}$ and finally high members of the Balmer series. This is due to the fact that continuum absorption coefficient increases toward longer wavelengths.

We temporarily exclude spectral lines in order to more conveniently trace how the continuous spectrum depends on the layer parameters. Figure 2 represents the ratio $I_{\lambda} / B_{\lambda}$ for two values of the electron temperature and three values of density. Atomic-ion temperature is equal here to $10 \mathrm{eV}$, and the thickness of the layer - to $10 \mathrm{~km}$. The values of the optical depth in a continuous spectrum at the wavelength $4170 \AA$ are shown on each curve. It is seen that increasing of the continuum opacity essentially depends on the electron temperature: at higher values of $T_{e}$, the transition to the Planck curve occurs at lower values of $N_{\mathrm{H}}$. This fact is caused by increasing of the occupation of excited levels $(k \geq 3)$ with increasing the electron temperature. Indeed, the value of $\varkappa_{\nu k}^{(i)} \propto N_{k}$, as it follows from equations (28) and (29b).

While the gas density is increased, the intensity of the continuous spectrum $I_{\lambda}$ tends to the Planck function, remaining less than it. The curve portions that are closer to the Planck curve are located in the red region of the spectrum and at the blue side of the Balmer jump. When the density increases further, the blue part of the spectrum (redder at the Balmer jump) approaches the Planck function.

The shape of the line core is largely determined by the ion-atom temperature, as the Doppler width, $\Delta \omega_{k n^{\prime}}^{D}$, depends only on the square root of $T_{a i}$ (if the principal quantum numbers $k$ and $n^{\prime}$ are unchanged). It is true that at higher values of $T_{a i}$, the approximation for the core by a gaussian curve is valid for a higher number of members of the Balmer series.

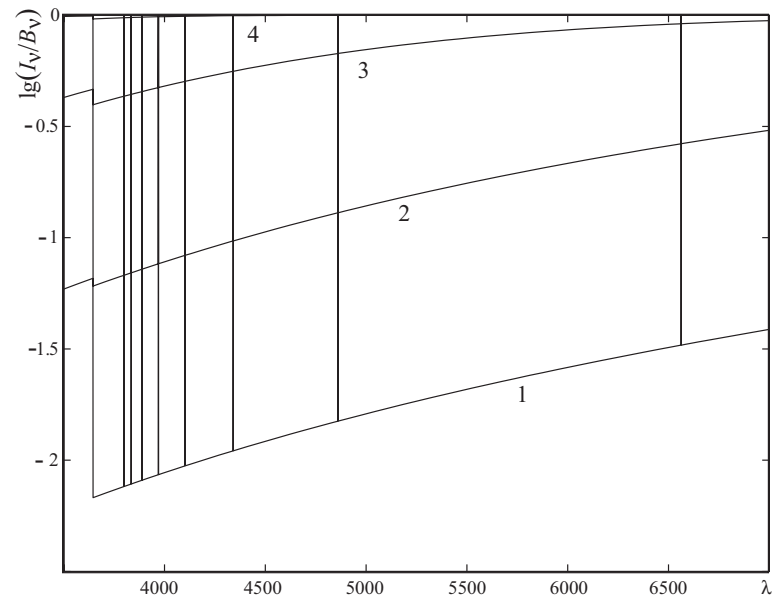

Fig. 1 The continuous spectrum and the height of the lines of the Balmer series for the four density values. From bottom up: $1 \cdot 10^{15} \mathrm{~cm}^{-3}, 3 \cdot 10^{15} \mathrm{~cm}^{-3}, 1 \cdot 10^{16} \mathrm{~cm}^{-3}$, $3 \cdot 10^{16} \mathrm{~cm}^{-3}$. Under each curve its number is written. The upper curve almost coincides with the Planck function; the continuum absorption is not negligible in this case.

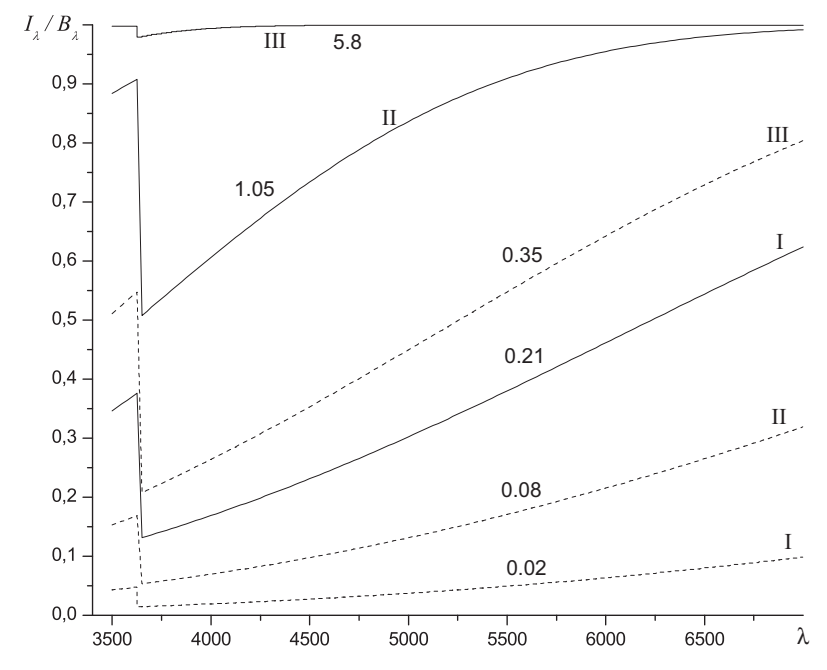

Fig. 2 The continuous spectrum normalized to the Planck function (spectral lines are excluded). The dashed lines correspond to $T_{e}=0.8 \mathrm{eV}$, solid ones - to $T_{e}=1.0 \mathrm{eV}$, Numbers I, II, and III correspond to the following values of the total number densities of the hydrogen particles: $2 \cdot 10^{15} \mathrm{~cm}^{-3}, 7 \cdot 10^{15} \mathrm{~cm}^{-3}$, and $3 \cdot 10^{16} \mathrm{~cm}^{-3}$, respectively. Next, the values of the optical depth at wavelength $\lambda=4170 \AA$ are shown for each curve.

Table 1 Decimal logarithms of the optical depth at the centers of the Balmer lines $\left(T_{e}=1.2 \mathrm{eV}, T_{a i}=6 \mathrm{eV}\right.$, and $\mathcal{L}=20 \mathrm{~km}$ )

\begin{tabular}{ccccc}
\hline $\log N$ & $\mathrm{H}_{\alpha}$ & $\mathrm{H}_{\beta}$ & $\mathrm{H}_{\gamma}$ & $\mathrm{H}_{\delta}$ \\
15.0 & 6.40 & 5.54 & 5.06 & 4.73 \\
15.5 & 6.90 & 6.04 & 5.56 & 5.23 \\
16.0 & 7.40 & 6.54 & 6.07 & 5.74 \\
16.5 & 7.90 & 7.04 & 6.57 & 6.24 \\
\hline
\end{tabular}




\section{Discussion}

The main result of our work is the calculated plasma emission spectrum in a broad interval of the continuum optical depths, from the transparent gas to the gas whose emission intensity is close to the Planck function. The proximity to the Planck function in the case of strong self-absorption is due to the mechanism of impact ionization and excitation (from electron collisions) of the plasma, whereas radiative processes are secondary. The approach used in this paper fits well for red dwarf stars with their effective temperature of about 3000 K. However, the case of G- and F-type dwarfs requires taking into account the photospheric emission, e.g., photoionization of hydrogen atoms from exited levels. In the case of appreciable emission and absorption in the continuum its (continuum) influence must be taken into account in the calculation of spectral line intensities. Here our work develops the approach used in Drake \& Ulrich (1980) and Katsova et al. (1991), where the line intensities were calculated under the assumption of the transparent continuum bringing small contribution to the emission.

In the framework of the considered problem the intensity of the subordinate lines is close to Planck for any layer parameters implemented during the flares on red dwarf stars. Therefore, the relative height of the lines depends on the optical depth in the continuum. If it is small then strong lines are observed against the background of faint continuum, which is described by formulae valid for transparent gas. In the case of appreciable self-absorption in the continuum its spectrum becomes close to Planck; thereby, the lines weaken. However, in the homogeneous layer model no strong lines on the background of the Planck continuum observed by Kowalski et al. (2013) are obtained. Probably, we see in the flares the total emission from several layers; some of them are transparent in the continuum, while other ones are not. We hope to get a better agreement with observations by calculations of the radiative cooling behind the shock front under the conditions of the red dwarf chromospheres, since the shocked gas can include at the same time equilibrium and non-equilibrium regions.

Table 2 Menzel factors for the discrete levels of the hydrogen atom

\begin{tabular}{cccccc}
\hline $\log N$ & 1 & 2 & 3 & 4 & 5 \\
15.0 & 1.030 & 1.031 & 1.006 & 1.001 & 1.000 \\
15.5 & 1.009 & 1.009 & 1.003 & 1.002 & 1.001 \\
16.0 & 1.003 & 1.003 & 1.002 & 1.001 & 1.000 \\
16.5 & 1.001 & 1.001 & 1.001 & 1.001 & 1.000 \\
\hline
\end{tabular}

\section{Acknowledgments}

E.S. Morchenko is grateful to Prof. Yu.K. Zemtsov for valuable remarks. This work was partly supported by the Russian Foundation for Basic Research (project code 14-02-00922), and the Scientific School (project code 1675.2014.2 NSh). We thank the anonymous referee for very useful suggestions and comments. 


\section{References}

Gershberg, R.E., Pikel'ner S.B.: Comments Astrophys. Space Phys. 4, 113 (1972).

Gershberg, R.E.: Solar-Type Activity in Main-Sequence Stars, (Springer) (2005).

Kostyuk, N.D., Pikel'ner, S.B.: Soviet Astron. 18, 590 (1975).

Katsova, M.M., Kosovichev, A.G., Livshits, M.A.: Astrophysics. 17, 156 (1981). doi: 10.1007/BF01005196

Fadeyev, Yu.A., Gillet, D.: Astron. Astrophys. 368, 901 (2001). arXiv:astro-ph/0603510 doi:10.1086/506268

Belova, O.M., Bychkov, K.V., Morchenko, E.S., Nizamov, B.A.: Astron. Reports. 58, 650 (2014) doi:10.1134/ S1063772914090029

Kowalski, A.F., Hawley, S.L., Wisniewski, J.P., Osten, R.A., Hilton, E.J., Holtzman, J.A., Schmidt, S.J., Davenport, J.R.A.: Astrophys. J. Suppl. Ser. 207, 57 (2013). 1307.2099. doi:10.1088/0067-0049/207/1/15

Drake, S.A., Ulrich, R.K.: Astrophys. J. Suppl. Ser. 42, 351, 1980.

Zel'dovich, Ya.B., Raizer, Yu.P.: Physics of Shock Waves and High-Temperature Hydrodynamic Phenomena, (Academic Press) (1967).

Johnson, L. C.: Astrophys. J. 174, 227 (1972).

Sobel'man, I.I.: Introduction to the Theory of Atomic Spectra, (Pergamon Press) (1972).

Vidal, C.R.: J. Quant. Spectrosc. Rad. Transfer 6, 461 (1966).

Holstein, T.: Phys. Rev. 72, 1212 (1947).

Biberman, L.M., Vorob'ev, V.S., Yakubov, I.T.: Kinetics of Nonequilibrium Low-Temperature Plasmas, (Plenum Publishers) (1987).

Smirnov, B.M.: Physics of weakly ionized gas, (Nauka) (1972).

Sobolev, V.V., Grinin V.P.: In: Pallavicini, R., Dupree, A.K. (eds.) Cool Stars, Stellar Systems, and the Sun, 9th Cambridge Workshop ASP Conference Series. 109, 629 (1996).

Sobolev, V.V., Grinin, V.P.: Astrophysics. 38, 15 (1995). doi:10.1007/BF02113956

Stehle, C.: Astron. Astrophys. Suppl. Ser. 104, 509 (1994).

Ivanov, V.V.: Transfer of Radiation in Spectral Lines, (U.S. Government Printing Office) (1973).

Mihalas, D.: Stellar Atmospheres, (W.H. Freeman and Co.) (1974).

Eason, E.L.E., Giampapa, M.S., Radick, R.R., Worden, S.P., Hege, E.K.: Astron. J. 104, 1161 (1992).

Underhill, A.B., Waddell, J.H.: National Bureau Standards Circular No. 603 (1959).

Katsova, M.M., Livshits, M.A., Butler, C.J., Doyle, J.G.: Mon. Not. R. Astron. Soc. 250, 402 (1991).

This manuscript was prepared with the AAS LATEX macros v5.2. 